\title{
Case of prosthetic valve endocarditis with osteomyelitis associated with disregarded skin infection
}

\author{
Michihito Nonaka*, Atsushi Iwakura and Kazuo Yamanaka \\ *Correspondence: mnonaka0725@hotmail.co.jp \\ Department of Cardiovascular Surgery, Tenri Hospital, 200, Mishima-cho, Tenri, Nara 632-8552, Japan.
}

\begin{abstract}
Background: Prosthetic valve endocarditis (PVE) is a serious condition associated with high mortality rate. The causes of PVE vary from surgical procedure to superficial skin infection. Sometimes it is difficult to identify latent infections in the body and recognize them as the true causes of PVE. We describe here a case of PVE complicated by osteomyelitis that was hematogenously disseminated from a disregarded infected skin tumor on the head.

Methods: A 73-year-old man who underwent aortic valve replacement developed prosthetic valve endocarditis complicated by osteomyelitis that was disseminated from an infected skin tumor on the head. During the follow-up after the first operation, neither a small skin mass on the head nor lower back pain had been considered manifestations of infection. With progressive pain and fever, however, the patient was referred back to our hospital for further examination; vegetation and paravalvular leakage around the aortic valve and lumbar osteomyelitis was detected.

Results: A Staphylococcus aureus blood culture confirmed the diagnosis of prosthetic valve endocarditis associated with osteomyelitis, originating from the infected skin tumor. Re-operation was performed. The affected aortic valve was excised through a transverse aortotomy, revealing a deep annular abscess and partial valvular dehiscence. After the abscess was debrided completely, the damaged annulus was reconstructed with an autologous pericardium. For the valve implantation, pledgeted 2-0 mattress sutures were threaded through the aortic root from the outside, considering the potential fragility of the annulus. With multidisciplinary treatment, re-aortic valve replacement by cardiac surgeons, removal of the skin tumor by dermatologists, and intensive antibiotic therapy for osteomyelitis by orthopedists, the patient recovered.
\end{abstract}

Conclusion: Valve replacement patients are vulnerable to external infective agents, hence awareness should be directed towards the potential threats.

Keywords: Endocarditis, prosthesis, infection, co-morbidity, reoperation

\section{Introduction}

Prosthetic valve endocarditis (PVE) is a serious condition associated with high mortality rate [1]. The causes of PVE vary from surgical procedure to superficial skin infection [2]. Since prosthetic valves are susceptible to external agents, patients after valve replacement are exposed to possible threats. Apart from dental caries, other regions of the body that are prone to infection, eg., the genitourinary tract, should be checked carefully to prevent PVE. However, sometimes it is difficult to identify latent infections in the body and recognize them as true causes of PVE. We describe here a case of PVE complicated by osteomyelitis that was hematogenously disseminated from a disregarded infected skin tumor on the head.

\section{Case presentation}

A 73-year-old man, who underwent aortic valve replacement (AVR), developed progressive back pain 9 months after the initial operation. Initially, considering it to be lumbar spondylosis, it was treated with an analgesic. Eventually, the inflammation increased such that the white blood cell count was $12,000 / \mu \mathrm{l}$ and the C-reactive protein level was $8.5 \mathrm{mg} / \mathrm{dl}$. Echocardiography revealed the presence of a small vegetation and paravalvular leakage around the aortic valve, and blood culture was found positive for Staphylococcus aureus. Thus, PVE was diagnosed. In addition, examination by computed tomography (CT) and magnetic resonance imaging (MRI) showed deformity of the L4 and L5 lumbar spine (Figure 1). Orthopedists suggested the presence of lumbar osteomyelitis, making surgical removal difficult. Moreover, further physical examination discovered a skin tumor on the head, upon which an ulcer had formed. Although the tumor was identified as benign, S. aureus was detected in the serum isolated from the ulcer. Therefore, it was deduced that infection first developed in the ulcer on the head, and then hematogenously disseminated to the spine, where it caused osteomyelitis; the infection had simultaneously extended to the prosthetic valve too.

After obtaining informed consent from the patient, re-AVR with a bioprosthetic valve was performed, followed by resection of the skin tumor by dermatologists. After careful dissection around the heart, cardiopulmonary bypass (CPB) was established. The affected aortic valve was excised through a transverse aortotomy, revealing a deep annular abscess and partial valvular dehiscence (Figure 2). After the abscess was debrided completely, the damaged annulus was reconstructed with an 

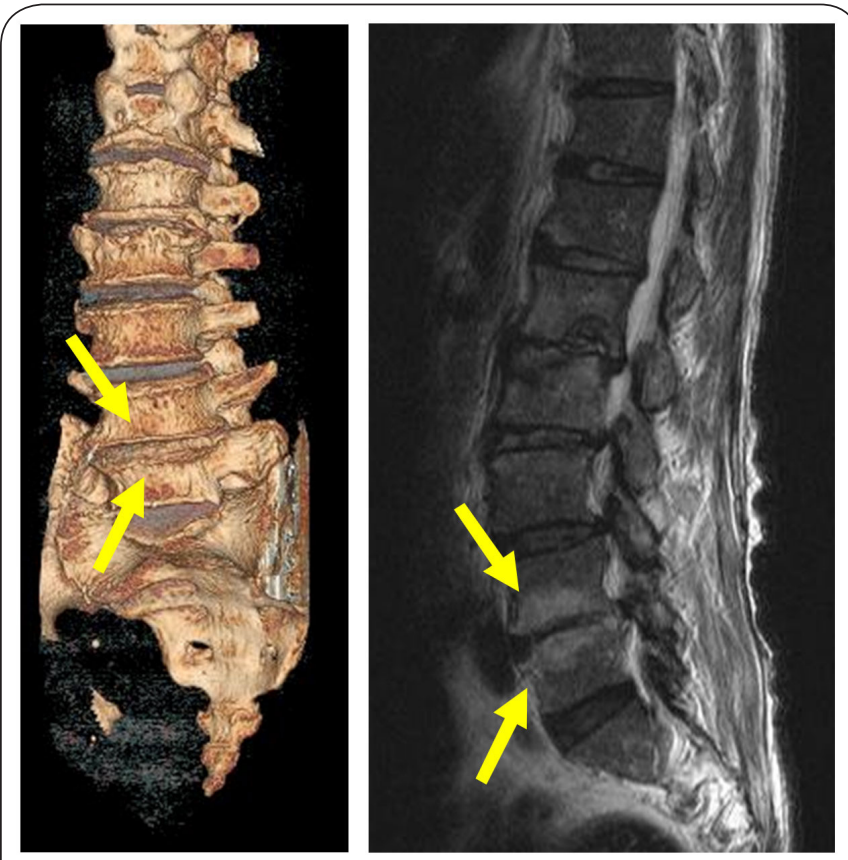

Figure 1. Computed tomography and magnetic resonance imaging showing deformity of the L4 and L5 lumbar spine compatible with the finding of osteomyelitis.

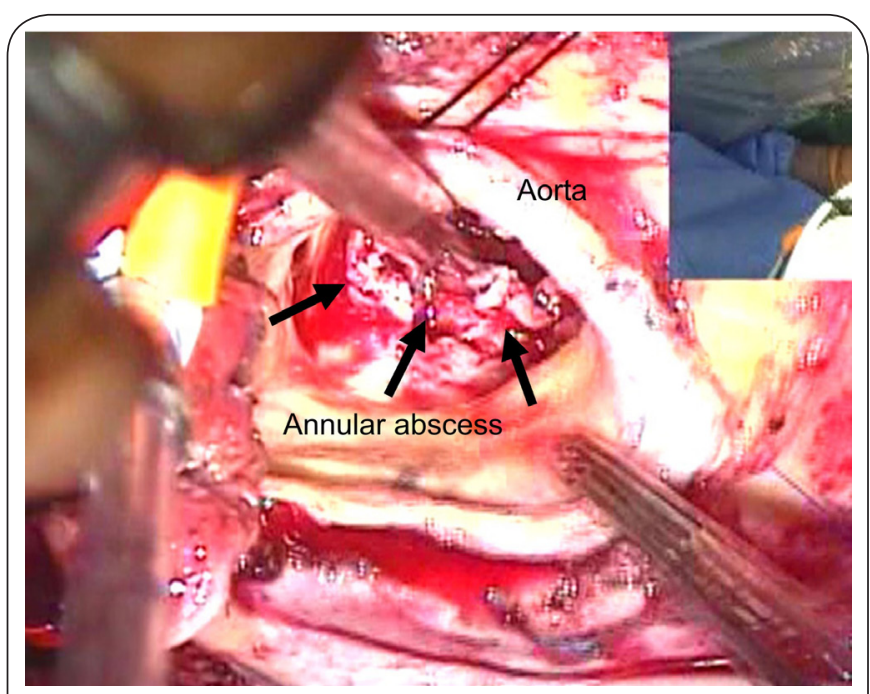

Figure 2. Operative findings demonstrating an abscess on the aortic annulus and partial valvular dehiscence.

autologous pericardium. For the valve implantation, mattress sutures of pledgeted 2-0 Ti-Cron (Ethicon, Massachusetts) were threaded through the aortic root from the outside, considering the potential fragility of the annulus. The heart was weaned from the CPB with stable hemodynamics, and hemostasis was achieved easily. After closing the chest, the head tumor was removed along with some surrounding tissue, and an autologous skin graft from the thigh was implanted to cover the area on the head that was operated on.

The patient woke up without complications and was extubated on the following day. As an antibiotic regimen for PVE and lumbar osteomyelitis, $2 \mathrm{~g}$ /day of vancomycin hydrochloride (VCM) was administered for 6 weeks. In addition, a lumbar harness was fitted to reduce the load to the affected spine. The infection cleared gradually and the tests for infection were negative at the time of discharge. Postoperative CT showed scarring of the affected areas of the lumbar spines. Through collaboration with other specialties, the patient recovered, and no signs of recurrent infection were observed over the 2-year follow-up.

\section{Discussion}

Prosthetic valve endocarditis, which has a high mortality rate of up to $60 \%$, is a serious complication with an incidence of $1-4 \%$ after valve surgery $[\mathbf{1}, \mathbf{3}]$. While dental procedure is recognized as the most common cause of PVE, other ordinary conditions, eg., acne vulgaris, which can cause systemic infection, are often overlooked [4]. Regarding this, Clayton et al., reported that skin infection is a risk factor for infective endocarditis [5]. Despite common concerns about infection, the skin region in this case was overlooked, thus predisposing the patient to PVE. If skin infection is more recognized as a potential threat for patients after valve replacement, the incidence of PVE cases like this will decrease. Therefore, greater awareness for potential skin infection is required for the follow-up after valve surgery.

Orthopedic diseases, especially spondylodiscitis and osteomyelitis, are often associated with infective endocarditis with a prevalence of $3.7-15 \%$ [6]. They are usually disseminated hematogeneously from other regions like decayed teeth. Since lower back pain is common in elder patients, it may not immediately be considered a manifestation of infection; however, MRI was effective in identifying the otherwise hidden infected area in this case. As another alternative, Thomson et al., [7] reported the usefulness of Gallium SPECT, with which they detected increased activity that indicated infection of the lumbar spine, as well as the mid-mediastinum, in cases of PVE. Orthopedic diseases accompanied by serious pain should be examined carefully without indiscreet administration of analgesic, which would mask fever and inflammatory reaction that might be caused by PVE.

Regarding treatment, it was reasonable that the skin tumor of the head was resected simultaneously with the reAVR. However, it is difficult to treat a region that cannot be removed-a lumbar spine in the present case-as the infected part remains in the body. As a treatment for lumbar osteomyelitis, conservative measures-the administration of antibiotics for 6 weeks and hyperbaric oxygenation, if available-are usually applied, although surgical procedure is undertaken for cases that show neurological deficits [8]. In the present case, according to the antimicrobial susceptibility of the causative microorganism, VCM was administered intravenously for 6 
Nonaka et al. Research Journal of Infectious Diseases 2013,

weeks, following the guideline for the treatment of PVE and osteomyelitis. In addition to the anti-biotic administration, the lumbar harness was effective for osteomyelitis because infection was alleviated by reducing the load to the spine and stabilizing the body.

\section{Conclusion}

We encountered a case of PVE complicated by osteomyelitis that was disseminated from a skin infection. Since patients who undergo valve replacement are susceptible to external infectious agents, it is vital to direct more attention not only to obvious surgical intervention but also to the superficial conditions that could be sources of infection.

\section{List of abbreviations}

AVR: Aortic valve replacement

CPB: Cardiopulmonary bypass

$\mathrm{CT}$ : Computed tomography

MRI: Magnetic resonance imaging

PVE: Prosthetic valve endocarditis

VCM: Vancomycin hydrochloride

\section{Competing interests}

The authors declare that they have no competing interests.

\section{Authors' contributions}

MN Conceived, planned, and performed the work leading to the report, or interpreted the evidence presented, or both, written the report or reviewed successive versions and participated in their revision. Al Approved the final version. KY Approved the final version.

\section{Publication history}

Editor: Isaac Karimi, Razi university, Iran.

Received: 20-May-2013 Revised: 11-Jul-2013

Accepted: 14-Jul-2013 Published: 27-Jul-2013

\section{References}

1. Ivert TS, Dismukes WE, Cobbs CG, Blackstone EH, Kirklin JW and Bergdahl LA. Prosthetic valve endocarditis. Circulation. 1984; 69:223-32. | Article I PubMed

2. Bayliss R, Clarke C, Oakley CM, Somerville W, Whitfield AG and Young SE. The microbiology and pathogenesis of infective endocarditis. Br Heart J. 1983; 50:513-9. | Article | PubMed Abstract | PubMed Full Text

3. Cowgill LD, Addonizio VP, Hopeman AR and Harken AH. A practical approach to prosthetic valve endocarditis. Ann Thorac Surg. 1987; 43:450-7. | Article | PubMed

4. Yamamoto T, Yodogawa K, Wakita S, Ogano M, Tokita M, Miyagi Y, Sato N, Nitta T, Tanaka $K$ and Takano T. Recurrent prosthetic valve endocarditis caused by Staphylococcus aureus colonizing skin lesions in severe atopic dermatitis. Intern Med. 2007; 46:571-3. | Article | PubMed

5. Clayton JJ, Baig W, Reynolds GW and Sandoe JA. Endocarditis caused by Propionibacterium species: a report of three cases and a review of clinical features and diagnostic difficulties. J Med Microbiol. 2006; 55:981-7. | Article | PubMed

6. Le Moal G, Roblot F, Paccalin M, Sosner P, Burucoa C, Roblot P and Becq-Giraudon B. Clinical and laboratory characteristics of infective endocarditis when associated with spondylodiscitis. Eur J Clin Microbiol Infect Dis. 2002; 21:671-5. | Article | PubMed

7. Thomson LE, Goodman MP, Naqvi TZ, Feldman R, Buchbinder NA,

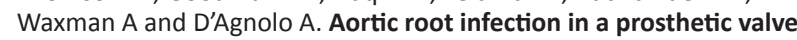
demonstrated by gallium-67 citrate SPECT. Clin Nucl Med. 2005; 30:2658. | Article | PubMed
8. Hadjipavlou AG, Mader JT, Necessary JT and Muffoletto AJ. Hematogenous pyogenic spinal infections and their surgical management. Spine (Phila Pa 1976). 2000; 25:1668-79. | Article | PubMed

\section{Citation:}

Nonaka M, Iwakura A and Yamanaka K. Case of prosthetic valve endocarditis with osteomyelitis associated with disregarded skin infection. Res J Infect Dis. 2013; 1:4. http://dx.doi.org/10.7243/2052-5958-1-4 